\title{
Type 2 diabetes and psoriasis: links and risks
}

This article was published in the following Dove Medical Press journal: Psoriasis:Targets and Therapy

\section{Jesper Grønlund Holm'} Simon Francis Thomsen ${ }^{1,2}$

'Department of DermatoVenereology, Bispebjerg Hospital, University of Copenhagen, Copenhagen, Denmark; ${ }^{2}$ Department of Biomedical Sciences, Faculty of Health and Medical Sciences, University of Copenhagen, Copenhagen, Denmark
Correspondence: Jesper Grønlund Holm Department of Dermatology, Bispebjerg Hospital, DK-2400 Copenhagen NV, Denmark

Tel +45 2637 I525

Email jespergholm@gmail.dk

\begin{abstract}
Psoriasis (PsO) is one of the most common chronic inflammatory skin diseases with a world prevalence of $2 \%-4 \%$. The increasing knowledge of the mechanisms driving $\mathrm{PsO}$ has raised focus on existing links to metabolic syndrome and type 2 diabetes (T2D). We reviewed the existing literature of the prevalence and risk of T2D in patients with PsO. The studies reviewed were mainly large retrospective cohort and case-control studies, showing an increased prevalence of T2D in PsO patients compared to controls, particularly in late onset (type 2) PsO. T2D prevalence did not correlate to patient age or severity of $\mathrm{PsO}$ in the reviewed studies. Conclusively, T2D was found to be more prevalent in patients with PsO compared to the background population. Several mechanisms involved in lipid transportation seem to be upregulated in $\mathrm{PsO}$ patients. Physicians play a key role concerning information about known comorbidity and promotion of early prophylaxis in patients with $\mathrm{PsO}$.
\end{abstract}

Keywords: psoriasis, type 2 diabetes, association, risk, link

\section{Introduction}

Psoriasis ( $\mathrm{PsO}$ ) is a chronic inflammatory skin disease that affects $2 \%-4 \%$ of the global population. ${ }^{1}$ The understanding of $\mathrm{PsO}$ as a systemic inflammatory disease along with the increasing knowledge of the mechanisms driving $\mathrm{PsO}$ has raised focus on existing links to metabolic syndrome and type 2 diabetes (T2D). ${ }^{2}$ The association of $\mathrm{PsO}$ with inflammatory and metabolic diseases has been investigated in numerous cohort studies. In addition, earlier reviews of the literature have assessed the association between $\mathrm{PsO}$ and T2D. ${ }^{3,4}$ However, the nature of the association between $\mathrm{PsO}$ and T2D is still ambiguous. In many studies it is unclear which disease came first, PsO or T2D. In addition, earlier studies did not group patients based on disease severity, which may have let to unclear conclusions about the true association between PsO and T2D. Herein we review studies examining the epidemiology of T2D in patients with PsO. Furthermore, we explore the possible pathophysiological links between PsO and T2D.

\section{Study selection}

We performed a search in PubMed using the keywords "psoriasis," "diabetes," "risk," "link," "association." Inclusion criteria were original, human studies written in English, focusing on the prevalence or risk of T2D in patients with PsO. Included study types were case reports, clinical studies, clinical trials, comparative studies, controlled clinical trials, multicenter studies, and randomized controlled trials. Exclusion criteria were animal studies, reviews, meta-analyses, comments on earlier publications, and studies not focusing on the direct relationship between $\mathrm{PsO}$ and T2D. Abstracts were read to

Psoriasis:Targets and Therapy 2019:9 I-6

Dovepress f 1 in $\nabla$

(c) (i) (5) 2019 Holm and Thomsen. This work is published and licensed by Dove Medical Press Limited. The full terms of this license are available at https://www.dovepress.com/terms. you hereby accept the Terms. Non-commercial uses of the work are permitted without any further permission from Dove Medical Press limited, provided the work is properly attributed. For
york permission for commercial use of this work, please see paragraphs 4.2 and 5 of our Terms (https.//www.dovepress.com/terms.php). 
evaluate the eligibility of the study. We intended to perform a complete and exhaustive review of the literature on T2D prevalence in $\mathrm{PsO}$ patients, focusing on major cohort studies.

The search in PubMed yielded a total of 1,357 hits. Exclusion of studies not written in English, animal studies, earlier reviews, and meta-analyses yielded 172 studies. All abstracts were evaluated, and 44 articles and abstracts were included (Supplementary material). The studies were published between 1995 and 2018 and included case-control studies $(25 / 44,56.8 \%)$, retrospective (14) and prospective (4) cohort studies $(18 / 44,40.9 \%)$, and a case series $(1 / 44$, $2.3 \%$ ). We divided and described the studies based on their focus; epidemiological studies focusing on T2D and other comorbidities in $\mathrm{PsO}$ patients (40 studies), where some described pathophysiological features of PsO subtypes (7/40 studies), and mechanistic and genetic links between PsO and T2D (four studies).

\section{$\mathrm{T} 2 \mathrm{D}$ in $\mathrm{PsO}$ patients}

A total of 15 studies were descriptive epidemiological cohorts, which included only $\mathrm{PsO}$ patients. The studies included on average 2,695 patients (range: $82-11,900$ ) from ten different countries (Brazil, ${ }^{5}$ Italy, ${ }^{6}$ France, ${ }^{7,8}$ Spain,,${ }^{9,10}$ Thailand, ${ }^{11}$ United Kingdom, ${ }^{12}$ Czech Republic, ${ }^{13}$ China, ${ }^{14}$ Turkey, ${ }^{15}$ and Romania ${ }^{16}$ ). Kwa et al ${ }^{17}$ (USA) who used population registry-based admissions as a proxy measure for $\mathrm{PsO}$ was excluded from the analysis. The average prevalence of T2D across $\mathrm{PsO}$ cohorts was $11.6 \%$, excluding Mihai et $\mathrm{al}^{16}$ due to an inaccurate T2D prevalence measure. ${ }^{16}$ Kimball et al, ${ }^{18}$ who reported baseline disease characteristics in a prospective PsO cohort including 11,900 patients, was the largest non-population-based study included. They found a T2D prevalence of $11.4 \%$, which agrees with the estimated average prevalence. Gisondi et $\mathrm{al}^{6}$ only found a T2D prevalence of $7 \%$ in a prospective interventional study, including 10,539 PsO patients, whereas Baeta et $\mathrm{al}^{5}$ performed a crosssectional study on 190 Brazilian PsO patients and found a T2D prevalence of $15.4 \%$, that is, three times that of the Brazilian background population. There was no apparent association between T2D prevalence and mean patient age $(\mathrm{n}=15, P=0.183)$ across the identified cohorts.

\section{T2D in PsO patients compared with controls}

The prevalence of T2D in PsO patients compared to controls was examined in eleven studies. All the eleven studies showed an increased prevalence of T2D compared to controls. Four studies used different outcomes, including impaired glucose tolerance, ${ }^{19}$ microvascular affection, ${ }^{20}$ waist-to-height ratio, ${ }^{21}$ and the risk of $\mathrm{PsO}$ in T2D patients. ${ }^{22}$ The studies included a median of 906 (range: 40-48,523, mean 5,579) patients and 1,230 (range: 40-208,187, mean: 24,788) controls. The mean T2D prevalence was $10.3 \%$ in patients and $6.2 \%$ in controls. Of note, Parisi et $\mathrm{al}^{23}$ compared 48,523 $\mathrm{PsO}$ patients with 208,187 controls in a large case-control study and found a slightly increased prevalence of T2D in PsO patients compared to controls $(5.78 \%$ vs $4.69 \%)$. Other studies showed a significant difference in T2D prevalence between cases and controls; however, a large variation in prevalence was observed between studies. Bang et $\mathrm{al}^{24}$ reported T2D in $18.1 \%$ of patients and $12.3 \%$ of controls, whereas Sommer et $\mathrm{al}^{25}$ reported $\mathrm{T} 2 \mathrm{D}$ in $11.7 \%$ of patients and $5.8 \%$ of controls, both in large cohorts. The heterogeneous findings complicate a reliable estimate of the true prevalence of T2D in patients with $\mathrm{PsO}$ and controls but support a significantly increased prevalence of T2D in $\mathrm{PsO}$ patients compared to healthy individuals. Ucak et a ${ }^{19}$ used oral glucose tolerance test instead of a diagnosis of T2D to estimate this association showing significantly impaired glucose tolerance in $\mathrm{PsO}$ patients, which offers a more objective para-clinical measure when comparing cohorts.

\section{$\mathrm{T} 2 \mathrm{D}$ and $\mathrm{PsO}$ severity}

Earlier studies have suggested that T2D prevalence may be correlated to the severity of PsO, suggesting that lack of stratification by severity may have impacted the conclusions in earlier studies on the association between the two conditions. However, across the PsO cohorts included herein, we found no evidence supporting a correlation between T2D prevalence and mean Psoriasis Area and Severity Index (PASI) $(n=5$, $P=0.188$ ), suggesting that $\mathrm{PsO}$ is a marker for increased risk of T2D independently of its severity. Despite this finding, Lee et $\mathrm{al}^{26}$ described that, in addition to an overall increased risk of T2D in $\mathrm{PsO}$ patients, the risk is modulated by both PsO severity, comorbidities and concomitant medication in a large Taiwan cohort. Also, while overt T2D is seemingly not related to severity of $\mathrm{PsO}$, intermediate phenotypes underlying the risk of T2D such as insulin resistance, fasting blood glucose and hemoglobin A1C may still be associated with the severity of $\mathrm{PsO} .{ }^{27} \mathrm{Naldi}$ et $\mathrm{al}^{28}$ concluded that the association found between $\mathrm{PsO}$ and T2D in other studies might have been confounded by lifestyle, smoking, overweight, or long disease duration, somewhat disagreeing with Lee et al. ${ }^{26}$ However, Mahé et $\mathrm{al}^{29}$ showed that patients with adult onset PsO had significantly more comorbidities compared to childhood onset patients. Therefore, long duration of disease may 
not be a risk factor as much as lifestyle. Supporting these findings, Karoli et $\mathrm{al}^{30}$ suggest that otherwise healthy $\mathrm{PsO}$ patients should take preventive measure toward modifiable risk factors, since the prevalence of systemic comorbidities is higher regardless of age of onset, however, associated with lifestyle choices.

\section{T2D in patients with $\mathrm{PsO}$ and psoriatic arthritis (PsA)}

Concomitant PsA is seen in up to $30 \%{ }^{31}$ of $\mathrm{PsO}$ patients and represents a systemic affection of psoriatic inflammation. This inflammatory upregulation possibly characterizes part of the metabolic component that links PsO to increased risk of T2D. In a large case-control study Dubreuil et $\mathrm{al}^{32}$ showed that $\mathrm{PsO}$ and $\mathrm{PsA}$ are both associated with risk of diabetes when adjusted for obesity and lifestyle factors, which agrees with Solomon et $\mathrm{al}^{33}$ who found an increased incidence rate of T2D in PsO + PsA as well as rheumatoid arthritis (RA) patients compared to controls, supporting the systemic nature of PsO. Despite the lack of correlation between $\mathrm{PsO}$ severity and risk of T2D, a study by Edson-Heredia et $\mathrm{al}^{34}$ found that patients with mild $\mathrm{PsO}$ were less affected by comorbid conditions than severe $\mathrm{PsO}$ patients. In addition, PsO patients were generally less affected than PsA patients. However, several studies showed no difference in the risk or prevalence of T2D in patients with PsA + PsO compared to patients with $\mathrm{PsO}$ alone. ${ }^{35,36}$ Dubreuil et $\mathrm{al}^{32}$ found that increased risk of diabetes in PsA was partially explained by obesity and lifestyle factors (alcohol and smoking) and that PsA and $\mathrm{PsO}$ were associated with the risk of diabetes when adjusted for obesity and lifestyle factors. This supports that the presence of PsA in patients with $\mathrm{PsO}$ mainly indicates a surplus of systemic inflammatory involvement, since autoimmune disorder prevalence, including T1D, does not differ between $\mathrm{PsO}$ and $\mathrm{PsO}+\mathrm{PsA}$ patients. ${ }^{36}$ Husted et $\mathrm{al}^{36}$ also suggest that increased cardiovascular (CV) risk in patients with PsA is mainly attributable to hypertension and not diabetes. A certain overlap may exist and supposedly studies need to consider a larger number of related conditions with relevance to the metabolic syndrome and $\mathrm{CV}$ disease to properly assess the correlation with $\mathrm{PsO}$.

\section{Other metabolic complications in $\mathrm{PsO}$ patients}

$\mathrm{PsO}$ is associated with several other metabolic complications, including hypertension, the metabolic syndrome, and overt heart disease. Understanding these associations play a significant role in optimizing treatment, comorbidity prophylaxis, and patient education. Karoli et al ${ }^{30}$ compared $96 \mathrm{PsO}$ patients with 100 controls and found that the prevalence of comorbidities associated with the metabolic syndrome (T2D, hyperlipidemia, and hypertension), including diagnosis of metabolic syndrome as a whole, was significantly increased in $\mathrm{PsO}$ patients (40\% vs 22\%). ${ }^{30}$ Agreeing with these findings, Phan et $\mathrm{al}^{7}$ found increased prevalence of hypertension, $\mathrm{T} 2 \mathrm{D}$, and dyslipidemia in French $\mathrm{PsO}$ patients compared to the general population. Karoli et $\mathrm{al}^{30}$ suggested preventive measures in otherwise healthy PsO patients, whereas Wan et $\mathrm{al}^{37}$ only suggested T2D prevention efforts in $\mathrm{PsO}$ patients with a body surface area of $\mathrm{PsO}>10 \%$. However, Parisi et $\mathrm{al}^{23}$ found that despite significantly higher BMI in patients with $\mathrm{PsO}$ compared to controls, $\mathrm{PsO}$ was not associated with $\mathrm{CV}$ events, when adjusting for comorbidities.

Bang et $\mathrm{al}^{24}$ state that $\mathrm{PsO}$ predicts new-onset atrial fibrillation, proposing how studies should delineate this association and determine the need for screening. However, Parisi et $\mathrm{al}^{23}$ found neither $\mathrm{PsO}$ nor severe $\mathrm{PsO}$ to be associated with risk of major $\mathrm{CV}$ events over 3-5 years after adjusting for known CV risk factors. ${ }^{23}$ Whether this is due to a relatively short follow-up period is unclear, but Mehta et $\mathrm{al}^{38}$ support the $\mathrm{CV}$ risk by a significantly more prevalent history of stroke and myocardial infarction in patients with $\mathrm{PsO}$ compared to controls. In addition, Karoli et $\mathrm{al}^{30}$ found that carotid thickness was associated with PsO, hypertension, T2D, and insulin resistance, all comprising the metabolic syndrome. This supports the presence of not only $\mathrm{CV}$ risk factors in $\mathrm{PsO}$ patients but also clinically measurable $\mathrm{CV}$ disease. Given that $\mathrm{PsO}$ manifests as a multisystem disease, Baeta et $\mathrm{al}^{5}$ suggest a comprehensive and multidisciplinary approach. The documented association with CV comorbidities may occur due to various factors such as the chronic inflammatory state of the disease, genetic susceptibility, and environmental factors and/or related to the quality of life or effects of drugs used for systemic therapy. Thus, prevention of risk factors associated with CV disease, especially in patients with severe disease and long disease duration, is recommended. ${ }^{15,39}$ In addition, Gisondi et $\mathrm{al}^{6}$ stress the importance of how choice of treatment should be weighed against the benefit and risks for each patient to ensure optimal management of symptoms and minimize acute and cumulative toxicities.

\section{T2D and sub-types of PsO}

Non-pustular PsO can be categorized as type 1 or type 2 based on the age at onset (early or late-onset). ${ }^{40}$ These subtypes and their respective immunological profiles as well as their differ- 
ent risks of T2D have been examined in seven of the included studies. Henseler and Christophers ${ }^{40}$ compared almost 3,000 $\mathrm{PsO}$ patients with other dermatological inpatients and found that diabetes, as well as obesity and heart failure, was more frequent in $\mathrm{PsO}$ compared to age-matched controls. In addition, they found increased resistance to cutaneous bacterial infections in early onset $\mathrm{PsO}$ patients and that cutaneous immune disorders such as contact and atopic dermatitis (AD) and urticaria are underrepresented in $\mathrm{PsO}$ patients, compared to control subjects with other dermatological conditions. They proposed that this resistance to infection was due to Cw6-haplotype (type 1, early onset). This agrees with the findings by Christophers ${ }^{41}$ who found that early onset $\mathrm{PsO}$ was dominated by certain leukocyte antigens (Cw6, B13, and $\mathrm{Bw} 57)$, whereas late-onset $\mathrm{PsO}$ showed a different profile (Cw2 and B27) suggesting that PsO, immunologically, is not a single disease. Both Sommer et $\mathrm{al}^{25}$ and Mahé et $\mathrm{al}^{29}$ showed greater risk of T2D in type $2 \mathrm{PsO}$ compared to type 1 , and Ucak et al $^{19}$ showed impaired glucose tolerance mainly in type $2 \mathrm{PsO}$ patients. Phan et $\mathrm{al}^{8}$ found that early onset of PsO in the elderly was associated with comorbidities. Theodorakopoulou et $\mathrm{al}^{42}$ found type $2 \mathrm{PsO}$ to be associated with T2D. Lastly, $\mathrm{Xu}$ et $\mathrm{al}^{14}$ found that metabolic disorders related to lipids were predominant in T1D, whereas type 2 $\mathrm{PsO}$ showed higher prevalence of T2D. Type 1 and type $2 \mathrm{PsO}$ show certain differences with T2D prevalence as the most apparent. Further research is warranted on the immunological diversities between PsO subtypes.

The similarities and differences between $\mathrm{PsO}$ and related diseases have also been the focus of other studies. Radtke et $\mathrm{al}^{43}$ found several diseases including asthma, vitiligo, and attention deficit/hyperactivity disorder to be more prevalent in AD compared to $\mathrm{PsO}$, whereas diseases included in the metabolic syndrome were higher in $\mathrm{PsO}$ patients. These findings support newer pathomechanistic knowledge on the difference between $\mathrm{PsO}$ and $\mathrm{AD}$, where barrier protective interleukins are downregulated in $\mathrm{AD}$, but upregulated in $\mathrm{PsO}{ }^{44,45}$

\section{Mechanistic links between $\mathrm{PsO}$ and T2D}

Mechanistic and genetic links between PsO and T2D have been described in several smaller studies.

Cheung et $\mathrm{al}^{46}$ analyzed and compared miRNA in lesional with non-lesional skin in a small case series of seven $\mathrm{PsO}$ patients. They found miRNA related to cholesterol efflux upregulated in lesional skin, believed to serve as a mechanistic link between psoriatic skin inflammation and comorbidities. Levels of the lipid transportation protein clusterin was compared between 15 moderate-severe $\mathrm{PsO}$ patients and controls by Buquicchio et al, ${ }^{47}$ who found increased levels in PsO patients. Both the studies support how certain relevant metabolic mechanisms differ from healthy controls, though the significance of this is uncertain. The knowledge on what role these findings play in $\mathrm{PsO}$ and associated metabolic comorbidity is not yet understood, however, different studies agree that patients with $\mathrm{PsO}$ show upregulation of metabolites associated with increased $\mathrm{CV}$ risk. ${ }^{48}$

Theodorakopoulou et $\mathrm{al}^{42}$ compared type 1 to type $2 \mathrm{PsO}$ patients based on demographics and phenotypes and found an increased lymphocytic infiltration in T1D patients. In addition, they showed that CD4+:CD8+ lymphocyte ratio was highest in T2D patients. The immunological differences between subtypes warrant further research. Lastly, Mihai et $\mathrm{al}^{16}$ included 82 Chinese $\mathrm{PsO}$ patients for genetic expression analysis. They measured levels of a panel of single-nucleotide polymorphisms (SNPs) in cytokine encoding genes and compared the levels to disease severity, though without any significant findings. Still, two SNPs were associated with T2D, one of which has been associated with PsO and PsO severity in a Chinese cohort. ${ }^{49}$ They concluded that certain variants in cytokine encoding genes influence multiple organ systems, which should stimulate research about the development of metabolic syndrome in $\mathrm{PsO}$ patients.

The knowledge of the genetic mechanisms involved in systemic complications and comorbidities in $\mathrm{PsO}$ patients is sparse. Now when the pathomechanisms of PsO are described in increasingly more detail and with the disease being controlled well with biological therapies, the management of complications and comorbidities is the next challenge. Therefore, knowledge of mechanistic links between $\mathrm{PsO}$ and T2D should be investigated, focusing on possible therapeutic targets. In addition, continuous knowledge on PsO comorbidities are important to track and adjust preventive efforts.

\section{Conclusion}

It is well established that T2D is more prevalent in patients with $\mathrm{PsO}$ compared to the background population. However, the systemic inflammatory nature of $\mathrm{PsO}$ is believed to include multiple organ systems, accounting for the metabolic diseases associated with PsO. The exact mechanistic links between the two conditions are not entirely understood and warrant further research. However, an increasing amount of literature has investigated both metabolic and genetic links believed to connect these diseases. Physicians play a key role in concerning information regarding known comorbidity risk and promotion of early prophylaxis in patients with $\mathrm{PsO}$. 


\section{Disclosure}

The authors report no conflicts of interest in this work.

\section{References}

1. Nestle FO, Kaplan DH, Barker J. Psoriasis. N Engl J Med. 2009:361(5): 496-509.

2. Oliveira M de F, Rocha B de O, Duarte GV. Psoriasis: classical and emerging comorbidities. An Bras Dermatol. 2015;90(1):9-20.

3. Armstrong AW, Harskamp CT, Armstrong EJ. Psoriasis and the risk of diabetes mellitus. JAMA Dermatol. 2013;149(1):84-91.

4. Coto-Segura P, Eiris-Salvado N, González-Lara L, et al. Psoriasis, psoriatic arthritis and type 2 diabetes mellitus: a systematic review and meta-analysis. Br J Dermatol. 2013;169(4):783-793.

5. Baeta IG, Bittencourt FV, Gontijo B, Goulart EM. Comorbidities and cardiovascular risk factors in patients with psoriasis. An Bras Dermatol. 2014;89(5):735-744.

6. Gisondi P, Cazzaniga S, Chimenti S, et al; Psocare Study Group. Metabolic abnormalities associated with initiation of systemic treatment for psoriasis: evidence from the Italian Psocare registry. J Eur Acad Dermatology Venereol. 2013;27(1):e30-e41.

7. Phan C, Sigal ML, Lhafa M, et al; GEM Resopso. Metabolic comorbidities and hypertension in psoriasis patients in France. Comparisons with French national databases. Ann Dermatol Venereol. 2016;143(4):264-274.

8. Phan C, Sigal ML, Estève E, et al; GEM RESOPSO. Psoriasis in the elderly: epidemiological and clinical aspects, and evaluation of patients with very late onset psoriasis. J Eur Acad Dermatol Venereol. 2016;30(1):78-82.

9. Sanchez-Carazo JL, López-Estebaranz JL, Guisado C. Comorbidities and health-related quality of life in Spanish patients with moderate to severe psoriasis: a cross-sectional study (Arizona study). J Dermatol. 2014;41(8):673-678.

10. Vanaclocha F, Crespo-Erchiga V, Jiménez-Puya R, et al; Investigadores del estudio AQUILES. Immune-mediated inflammatory diseases and other comorbidities in patients with psoriasis: baseline characteristics of patients in the AQUILES study. Actas Dermosifiliogr. 2015;106(1):35-43 English, Spanish.

11. Pongpit J, Porntharukchareon S, Kaewduang P, et al. Liver stiffness measurement in psoriasis: do metabolic or disease factors play the important role? BioMed Research International. 2016;2016(5):1-6.

12. Dregan A, Chowienczyk P, Molokhia M. Cardiovascular and type 2 diabetes morbidity and all-cause mortality among diverse chronic inflammatory disorders. Heart. 2017;103(23):1867-1873.

13. Kojanova M, Fialova J, Cetkovska P, et al; BIOREP study group. Characteristics and risk profile of psoriasis patients included in the Czech national registry BIOREP and a comparison with other registries. Int $J$ Dermatol. 2017;56(4):428-434.

14. Xu X, Su L, Gao Y, Ding Y. The prevalence of nonalcoholic fatty liver disease and related metabolic comorbidities was associated with age at onset of moderate to severe plaque psoriasis: a cross-sectional study. PLoS One. 2017;12(1):e0169952.

15. Adışen E, Uzun S, Erduran F, Gürer MA. Prevalence of smoking, alcohol consumption and metabolic syndrome in patients with psoriasis. $A n$ Bras Dermatol. 2018;93(2):205-211.

16. Mihai VV, Iulia $S$, Alexandra $P$, et al. Gene polymorphisms of TNF238G / a, TNF-308G / a, IL10-1082G / a, TNFAIP3, and MC4R and comorbidity occurrence in a Romanian population with psoriasis. $J$ Med Life. 2018;11(1):69-74.

17. Kwa MC, Silverberg JI. Association between inflammatory skin disease and cardiovascular and cerebrovascular co-morbidities in US adults: analysis of nationwide inpatient sample data. Am J Clin Dermatol. 2017;18(6):813-823.

18. Kimball AB, Leonardi C, Stahle M, et al; PSOLAR Steering Committee. Demography, baseline disease characteristics and treatment history of patients with psoriasis enrolled in a multicentre, prospective, diseasebased registry (PSOLAR). Br J Dermatol. 2014;171(1):137-147.
19. Ucak S, Ekmekci T, Basat O, Koslu A, Altuntas Y. Comparison of various insulin sensivity indices in psoriatic patients and their relationship with type of psoriasis. J Eur Acad Dermatol Venerol. 2006;20(5):517-522.

20. Armstrong AW, Guérin A, Sundaram M, et al. Psoriasis and risk of diabetes-associated microvascular and macrovascular complications. J Am Acad Dermatol. 2015;72(6):968-977.e2.

21. Lee A, Smith SD, Hong E, Garnett S, Fischer G. Association between pediatric psoriasis and Waist-to-Height ratio in the absence of obesity: a multicenter Australian study. JAMA Dermatol. 2016;152(12):1314-1319.

22. Jacob L, Kostev K. Psoriasis risk in patients with type 2 diabetes in German primary care practices. Prim Care Diabetes. 2017;11(1):52-56.

23. Parisi R, Rutter MK, Lunt M, et al; Identification and Management of Psoriasis Associated ComorbidiTy (IMPACT) project team. Psoriasis and the risk of major cardiovascular events: cohort study using the clinical Practice Research Datalink. J Invest Dermatol. 2015;135(9):2189-2197.

24. Bang CN, Okin PM, Køber L, Wachtell K, Gottlieb AB, Devereux RB. Psoriasis is associated with subsequent atrial fibrillation in hypertensive patients with left ventricular hypertrophy: the losartan intervention for endpoint study. J Hypertens. 2014;32(3):667-672.

25. Sommer DM, Jenisch S, Suchan M, Christophers E, Weichenthal M Increased prevalence of the metabolic syndrome in patients with moderate to severe psoriasis. Arch Dermatol Res. 2006;298(7):321-328.

26. Lee MS, Lin RY, Lai MS. Increased risk of diabetes mellitus in relation to the severity of psoriasis, concomitant medication, and comorbidity: a nationwide population-based cohort study. J Am Acad Dermatol. 2014;70(4):691-698.

27. Polic M, Miskulin M, Smolic M, et al. Psoriasis severity—a risk factor of insulin resistance independent of metabolic syndrome. Int J Environ Res Public Health. 2018;15(7):E1486.

28. Naldi L, Chatenoud L, Belloni A, et al. Medical history, drug exposure and the risk of psoriasis. Evidence from an Italian case-control study. Dermatology. 2008;216(2):125-130; discussion 130-132.

29. Mahé E, Maccari F, Beauchet A, et al. Childhood-onset psoriasis: association with future cardiovascular and metabolic comorbidities Br J Dermatol. 2013;169(4):889-895.

30. Karoli R, Fatima J, Shukla V, et al. A study of cardio - metabolic risk profile in patients with psoriasis. JAssoc Physicians India. 2013;61(11):798-803.

31. Ritchlin CT, Colbert RA, Gladman DD, Arthritis P. Psoriatic arthritis N Engl J Med. 2017;376(10):957-970.

32. Dubreuil M, Rho YH, A M, Man A. Original article diabetes incidence in psoriatic arthritis, psoriasis and rheumatoid arthritis: a UK populationbased cohort study. Rheumatology (Oxford). 2014:53(2):346-352.

33. Solomon DH, Love TJ, Canning C, Schneeweiss S. Risk of diabetes among patients with rheumatoid arthritis, psoriatic arthritis and psoriasis. Ann Rheum Dis. 2010;69(12):2114-2117.

34. Edson-Heredia E, Zhu B, Lefevre C, et al. Prevalence and incidence rates of cardiovascular, autoimmune, and other diseases in patients with psoriatic or psoriatic arthritis: a retrospective study using clinical Practice Research Datalink. J Eur Acad Dermatol Venereol. 2015;29(5):955-963.

35. Nas K, Karkucak M, Durmus B, et al. Comorbidities in patients with psoriatic arthritis: a comparison with rheumatoid arthritis and psoriasis. Int J Rheum Dis. 2015;18(8):873-879.

36. Husted JA, Thavaneswaran A, Chandran V, et al. Cardiovascular and other comorbidities in patients with psoriatic arthritis: a comparison with patients with psoriasis. Arthritis Care Res (Hoboken). 2011;63(12):1729-1735.

37. Wan MT, Shin DB, Hubbard RA, Noe MH, Mehta NN, Gelfand JM. Psoriasis and the risk of diabetes: a prospective population-based cohort study. J Am Acad Dermatol. 2018;78(2):315-322.e1.

38. Mehta NN, Yu Y, Pinnelas R, et al. Attributable risk estimate of severe psoriasis on major cardiovascular events. Am J Med. 2011;124(8):775. e1-776.

39. Bostoen J, Van Praet L, Brochez L, Mielants H, Lambert J. A crosssectional study on the prevalence of metabolic syndrome in psoriasis compared to psoriatic arthritis. J Eur Acad Dermatol Venereol. 2014;28(4):507-511.

40. Henseler T, Christophers E. Disease concomitance in psoriasis. J Am Acad Dermatol. 1995;32(6):982-986. 
41. Christophers EHT. Patient subgroups and the inflammatory pattern in psoriasis. Acta Derm Venereol Suppl (Stockh). 1989;151:88-92; discussion $106-110$.

42. Theodorakopoulou E, Yiu ZZ, Bundy C, et al. Early- and late-onset psoriasis: a cross-sectional clinical and immunocytochemical investigation. Br J Dermatol. 2016;175(5):1038-1044.

43. Radtke MA, Schäfer I, Glaeske G, Jacobi A, Augustin M. Prevalence and comorbidities in adults with psoriasis compared to atopic eczema. J Eur Acad Dermatol Venereol. 2017;31(1):151-157.

44. Cho JS, Pietras EM, Garcia NC. IL-17 is essential for host defense against cutaneous Staphylococcus aureus infection in mice Find the latest version: IL-17 is essential for host defense against cutaneous Staphylococcus aureus infection in mice. 2010;120(5):1762-1773.

45. Czarnowicki T, Gonzalez J, Shemer A, et al. Severe atopic dermatitis is characterized by selective expansion of circulating TH2/TC2 and TH22/TC22, but not TH17/TC17, cells within the skin-homing T-cell population. J Allergy Clin Immunol. 2015;136(1):104-115.e7.
46. Cheung L, Fisher RM, Kuzmina N, et al. Psoriasis skin inflammationinduced microRNA-26b targets NCEH1 in underlying subcutaneous adipose tissue. J Invest Dermatol. 2016;136(3):640-648.

47. Buquicchio R, Foti C, Loconsole F, Polimeno L, Ventura MT. Clusterin serum level: how does it affect psoriatic patients? J Biol Regul Homeost Agents. 2017;31(3):785-789.

48. García-Rodríguez S, Arias-Santiago S, Perandrés-López R, et al. Decreased plasma levels of clusterin in patients with psoriasis. Actas Dermo-Sifiliográficas. 2013;104(6):497-503.

49. Zhang C, Zhu KJ, Liu H, et al. The TNFAIP3 polymorphism rs610604 both associates with the risk of psoriasis vulgaris and affects the clinical severity. Clin Exp Dermatol. 2015;40(4):426-430.
Psoriasis: Targets and Therapy

\section{Publish your work in this journal}

Psoriasis: Targets and Therapy is international, peer-reviewed, open access journal focusing on psoriasis, nail psoriasis, psoriatic arthritis and related conditions, identification of therapeutic targets and the optimal use of integrated treatment interventions to achieve improved outcomes

\section{Dovepress}

and quality of life. Visit http://www.dovepress.com/testimonials.php to read real quotes from published authors. 